\title{
Association of Bethesda category and molecular mutation in patients undergoing thyroidectomy
}

Marco Mascarella ${ }^{1}$, Magdalena Peeva ${ }^{2}$, Veronique-Isabelle Forest ${ }^{3}$, Marc Pusztaszeri ${ }^{3}$, Galit Avior ${ }^{4}$, Michael Tamilia ${ }^{3}$, Alex Mlynarek ${ }^{5}$, Michael Hier ${ }^{5}$, and Richard Payne ${ }^{5}$

${ }^{1}$ Research Institute of the McGill University Health Centre

${ }^{2}$ McGill University Faculty of Medicine

${ }^{3}$ Jewish General Hospital

${ }^{4}$ Hillel Yaffe Medical Center

${ }^{5}$ McGill University

March 21, 2021

\begin{abstract}
Objective: The aim of this study was to ascertain the relationship between Bethesda category and molecular mutation of thyroid nodules in patients undergoing thyroidectomy. Design: A retrospective cohort of patients who underwent thyroidectomy following needle biopsy and molecular profile testing was performed. Setting: Two tertiary care academic hospitals. Participants: Consecutive patients with a dominant thyroid nodule who underwent both USFNA and molecular profile testing followed by thyroidectomy were included in the study. Main Outcome and Measures: The main outcome was postoperative diagnosis of thyroid cancer and aggressivity of disease based on histopathological variants, nodal metastasis or extra-thyroidal extension. Associations between Bethesda category, molecular mutation and postoperative pathology was assessed using descriptive analysis and Chi-square testing. Results: 451 patients were included. 95.9\% (93/97) of patients with a BRAFV600E mutation had a Bethesda category V or VI $(\mathrm{P}<0.001)$, and all had confirmed thyroid cancer on postoperative pathology. Those with $\mathrm{H}$, $\mathrm{K}$ or N RAS or EIF1AX mutations, gene expression profiling (GEP) or copy number alterations showed an association with Bethesda categories III and IV (P[?]0.01). Those with no identified molecular mutation had a lower incidence of aggressive thyroid cancer compared to those with an identified mutation $(12.6 \%$ vs $44.3 \%, \mathrm{P}<0.01)$. Conclusion: BRAFV600E mutations were associated with thyroid cancer subtypes known to be more aggressive. These findings may help thyroid specialists better identify aggressive thyroid nodules associated with indeterminate Bethesda categories.
\end{abstract}

\section{ABSTRACT}

Objectives: The aim of this study was to ascertain the relationship between Bethesda category and molecular mutation of thyroid nodules in patients undergoing thyroidectomy.

Design: A retrospective cohort of patients who underwent thyroidectomy following needle biopsy and molecular profile testing was performed.

Setting: Two tertiary care academic hospitals.

Participants: Consecutive patients with a dominant thyroid nodule who underwent both USFNA and molecular profile testing followed by thyroidectomy were included in the study.

Main Outcome and Measures: The main outcome was postoperative diagnosis of thyroid cancer and aggressivity of disease based on histopathological variants, nodal metastasis or extra-thyroidal extension. Associations between Bethesda category, molecular mutation and postoperative pathology was assessed using descriptive analysis and Chi-square testing. 
Results: 451 patients were included. 95.9\% (93/97) of patients with a BRAFV600E mutation had a Bethesda category V or VI $(\mathrm{P}<0.001)$, and all had confirmed thyroid cancer on postoperative pathology. Those with H, K or N RAS or EIF1AX mutations, gene expression profiling (GEP) or copy number alterations showed an association with Bethesda categories III and IV (P[?]0.01). Those with no identified molecular mutation had a lower incidence of aggressive thyroid cancer compared to those with an identified mutation $(12.6 \%$ vs $44.3 \%, \mathrm{P}<0.01)$.

Conclusion: BRAFV600E mutations were associated with thyroid cancer subtypes known to be more aggressive. These findings may help thyroid specialists better identify aggressive thyroid nodules associated with indeterminate Bethesda categories.

\section{Key Points:}

Almost all patients with a BRAFV600E mutation have a Besthesda diagnostic category of V or VI. Patients with a RAS-type mutation were categorized as Bethesda III or IV. Preoperative molecular testing of dominant thyroid nodules may help guide management as patients with BRAFV600E mutations are likely to have more aggressive disease. Patients with no molecular mutation identified tended to have less aggressive thyroid cancer.

\section{INTRODUCTION}

The widespread use of medical imaging has contributed to the increased incidence of thyroid nodules detected and incidental thyroid cancer diagnosed. [1] Studies suggest that the number of ultrasound guided fine needles aspirations (USFNAs) performed has doubled and the number of thyroid surgeries performed has increased by nearly $30 \%$ over the last decade. [1] Currently, USFNA is the standard diagnostic method for thyroid nodules. Although USFNA specimens are categorized according to the Bethesda classification, $24 \%-57 \%$ of thyroid USFNAs have an indeterminate result. [2-4] As a consequence, thyroid lobectomy is often performed for diagnostic and potentially therapeutic purposes. In roughly $60 \%$ of patients with indeterminate cytology, the thyroid nodule is benign. [4] In these patients, molecular testing of thyroid nodules has become an adjunct to USFNA biopsy to reduce the likelihood of unnecessary diagnostic surgery. [5]

Mutations that are uncovered with molecular testing of thyroid nodules, namely BRAF V600E or RAS-type mutations, can assist with uncovering the phenotype of the nodule (benign versus malignant) as well as whether the tumor may be an aggressive variant. Mutations of the BRAF V600E gene, found in 35-70\% of papillary thyroid carcinomas (PTC), lead to the activation of BRAF kinase and chronically stimulate the MAPK signaling pathway. [7,8] The BRAF V600E mutation has been strongly associated with classic and tall cell variant of PTC. [8] This mutation has also been shown to correlate with aggressive tumor characteristics including multifocality, extrathyroidal extension, regional or distant metastases and an advanced tumor stage at presentation. [8-15] Moreover, studies have shown that BRAF V600E mutations are associated with tumor recurrence and poor prognosis. [14,15] Mutation in the three RAS genes (HRAS, KRAS, and NRAS) are found in follicular adenomas and follicular carcinomas, as well as in the follicular variant of PTC and more recently for non-invasive follicular neoplasm with papillary-like nuclear features (NIFTP). [7] RAS mutations result in permanent RAS activation which chronically stimulate the MAPK and PI3K/AKT signaling pathways. [7] They are associated follicular-patterned neoplasms, with less prominent nuclear features of PTC, more frequent encapsulation and a lower rate of lymph node metastases than those with BRAF V600E mutations. [16-18] According to a recent study, RAS mutations in malignant thyroid nodules, when isolated, are associated with low-risk features and a favorable prognosis. [16-18]

In this study, the association between Bethesda category and molecular mutation assessed with ThyroSeq V3 or ThyGeNEXT in patients with thyroid nodules undergoing thyroidectomy is explored to better tailor patient diagnosis and treatment.

\section{METHODS}

A retrospective multicenter cohort study of patients who underwent thyroid surgery at two tertiary care academic hospitals from January 1, 2016 to December 1, 2019 was performed. Consecutive patients with a 
dominant thyroid nodule who underwent both USFNA and molecular profile testing (ThyGeNEXT, Interpace Diagnostics, Parsippany, NJ, USA or ThyroSeq v3, UPMC, Pittsburgh, PA, USA) followed by thyroidectomy were included in the study. Indications for molecular profile testing of thyroid nodules was for cytologically indeterminate Bethesda category (III, IV, V) or discordance cytology results with ultrasound findings. Patients who did not undergo thyroidectomy as well as pediatric patients were excluded from the study. This study was approved by the Research Ethics Committee at the tertiary care center (REB 2019-1532).

Clinical data including age, sex, Bethesda category of the dominant nodule, and if a molecular mutation was identified were collected. In addition, the extent of thyroidectomy performed (hemi, total or completion thyroidectomy) and postoperative histopathology were noted. Postoperative histopathology was classified as benign disease, malignant or non-invasive follicular thyroid neoplasm with papillary-like nuclear features (NIFTP). Furthermore, PTC of less than $10 \mathrm{~mm}$ were classified as microcarcinomas. Additionally, aggressive thyroid cancer was defined as any evidence of thyroid carcinoma variants (tall cell, hobnail, solid, diffuse sclerosing, anaplastic or poorly differentiated), clinical evidence of extrathyroidal extension or central neck metastasis.

The STROBE guidelines were used for reporting data. Descriptive statistics to characterize the cohort was performed. The association between Bethesda category and type of molecular mutation of dominant thyroid nodule on USFNA was compared using Chi-square testing. Logistic regression was performed to quantify the measure of association between Bethesda category and type of molecular mutation of the dominant thyroid nodule. Also, comparison of Bethesda category and molecular mutation to postoperative pathology was performed using Chi-square testing.

\section{RESULTS}

A total of 451 patients with a thyroid nodule undergoing USFNA, molecular profile testing and surgery were included in the study. Baseline patient characteristics are shown in Table 1. Most patients were female and had a mean dominant thyroid nodule size of $2.1 \mathrm{~cm}$ (standard deviation of $1.1 \mathrm{~cm}$ ). 325 patients $(72.1 \%$ ) underwent thyroid lobectomy. The overall prevalence of malignancy, excluding cases of NIFTP, was $78.5 \%$ of the cohort. The prevalence of postoperative malignancy by USFNA Bethesda category was $56.7 \%$ (55/97) for Bethesda III, 75.7\% (109/144) for Bethesda IV, 80.1\% (86/104) for Bethesda V and 100\% for Bethesda VI lesions. 138 patients showed evidence of aggressive thyroid cancer of which 79 had central neck lymph node metastasis.

Select mutations of dominant thyroid nodules were strongly associated with specific Bethesda categories (Table 2). Almost all BRAF V600E mutations were associated with Bethesda V and VI $(\mathrm{P}<0.0001)$. Only 4 of $97(4.1 \%)$ nodules with BRAF V600E mutations had a Bethesda category III or IV, all of which were malignant on postoperative pathology. RAS and EIF1AX mutations, copy number alterations and GEP were strongly associated with Bethesda categories III and IV (P[?]0.01). Figure 1 illustrates the above findings by displaying the frequency of Bethesda categories among different molecular mutations. Thyroid nodules with an identified molecular mutation were more likely to harbour thyroid cancer. Specifically, nodules with a BRAF V600E, TERT or RET mutation were most likely to harbour an aggressive thyroid cancer (Table $3)$.

Other identified molecular mutations were less associated with aggressive disease. NIFTP was mostly associated with RAS-type mutations. No molecular mutations were identified in $43.9 \%$ of patients; of those patients, 128 had a thyroid cancer. Overall, those with no identified molecular mutation had a lower incidence of aggressive thyroid cancer compared to those with an identified mutation $(12.6 \%$ vs $44.3 \%, \mathrm{P}<0.01)$. When classified according to malignancy on postoperative pathology, statistically significant associations to Bethesda category by molecular mutation were identified in patients with BRAF V600E, RAS, PTEN and EIF1AX mutations, copy number alterations and GEP (Supplemental Table 1). The presence of an identified molecular mutation on USFNA predicted postoperative malignancy.

\section{DISCUSSION}


Our study shows that thyroid nodules with BRAF V600E mutations are strongly associated with Bethesda categories V and VI, whereas nodules with a RAS or EIF1AX mutation, copy number alteration or GEP are more commonly associated with Bethesda categories III and IV. Certain mutations within dominant thyroid nodules, specifically those with a BRAF V600E, TERT or RET mutation, were associated with thyroid cancer subtypes known to be more aggressive. An increasing number of studies have concluded that molecular testing of thyroid nodules has a significant diagnostic as well as prognostic value and can help improve management of patients. [19-25] Other studies, however, question the clinical utility and costbenefit analysis of molecular testing. [20,24] The Afirma Gene Expression Classifier (AfirmaßR, Veracyte, Inc., South San Francisco, CA) measures mRNA transcript levels of 142 genes and has shown to have a high sensitivity of $91 \%$ and a moderate specificity of $68 \%$. [18,22] ThyGeNEXT (ThyGeNEXTß, Interpace Diagnostics, PDI Inc, Parsippany, NJ) and ThyroSeq v3 (ThyroSeq® joint partnership between UPMC and CBLPath, Pittsburgh, PA) are two comprehensive tests, designed to improve the overall accuracy of molecular testing, with sensitivity of $89 \%$ and $94 \%$ and specificity of $85 \%$ and $82 \%$, respectively. [20,24] The data regarding the clinical utility of molecular testing in patients with a Bethesda $\mathrm{V}$ and VI thyroid nodules remains unclear. [24-28] The value in molecular testing for such thyroid nodules lies not in the fact that this tool can predict malignancy. Further characterizing Bethesda V and VI thyroid nodules with molecular testing can identify patients with higher-risk disease (ex. presence of BRAF V600E, TERT) and may offer clinicians better perioperative patient counselling and prognostication.

The American Association of Endocrine Surgeons 2020 guidelines strongly recommend molecular testing as a diagnostic adjunct for cytologically indeterminate thyroid nodules if the information gained will affect clinical care. [20] Molecular testing may help clinicians with the management of thyroid nodules by identifying the nature of indeterminate lesions with mutations highly specific for malignancy such as BRAF V600E, RET or TERT, leading to improved diagnostic accuracy and potential reduction in the number of completion thyroidectomies performed. [22-24] Moreover, the extent of surgery performed on patients with a cytologic diagnosis of Bethesda category V or VI is related to the risk of recurrence and determined by the tumor size, radiographic features, presence of adenopathy and contralateral thyroid disease. [20] In this study, $44 \%$ of patients with a Bethesda category V or VI nodule harboured a BRAF V600E mutation. Given the relatively high prevalence of this mutation in Bethesda V/VI lesions, identifying this mutation can stratify patients into a distinct higher risk group. [8-15,22] Preoperative knowledge of a BRAF V600E mutation can help clinicians recognize patients with high risk thyroid nodule and potentially offer personalized patient counselling and treatment planning, even in nodules of smaller size. In the four patients with a less than 2 $\mathrm{cm}$ Bethesda III/IV thyroid nodule, identification of a BRAF V600E mutation preoperatively allowed for a total thyroidectomy with elective neck dissection to be performed. All 4 patients showed aggressive thyroid cancer features: 2 with central neck metastasis and 2 with solid or tall cell variants of papillary thyroid cancer.

The management of patients with thyroid nodules that are indeterminate on cytology often consists of a diagnostic lobectomy or active surveillance based on clinical factors including the nodule's size, enlargement on ultrasound, suspicious ultrasound features, clinical and family history, history of radiation exposure, and patient's preferences. [5,20, 25] Yip et al. show that molecular testing of thyroid nodules can also be useful in defining the extent of surgery for patients with low risk mutations to thyroid lobectomy. [24] Understanding that RAS-type mutations, which are shown to have a favorable prognosis when isolated, are commonly associated with Bethesda III and IV suggests that patients with an isolated RAS mutation may potentially be more conservatively treated with lobectomy. [25] The current results show that BRAF V600E mutations have been identified in $4.1 \%$ of indeterminate nodules which highlights the need for molecular testing to identify nodules of higher risk of malignancy. In this context, it may be warranted for the thyroid specialist to consider mutation testing analysis for specific higher-risk mutations such as BRAF V600E, TERT or RET as a means to preoperatively risk stratify patients. That being said, 128 patients did not have an identified molecular mutation with $64.6 \%$ proving to be malignant. In this subgroup of patients, only $12.6 \%$ of patients showed evidence of aggressive variants of thyroid cancer or central neck metastasis and is significantly lower in prevalence compared to patients with an identified molecular mutation. Additional understanding of thyroid 
carcinogenesis, presence of co-mutations and heterogeneity of nodules is needed to better allow clinicians to predict risk of malignancy and tumor aggressivity. [25]

Certain limitations to this study are related to the retrospective design and inclusion of patients undergoing molecular testing and thyroidectomy. By doing so, the overall prevalence of malignancy was elevated due to the selection of cases with more suspicious disease. Also, the indication for molecular profile testing was at the surgeon's discretion. Expenses related to molecular profile testing of thyroid nodules is currently not covered in the Quebec health system; this may introduce a selection bias for patients who have the financial means to access it. Lastly, validation with larger prospectively collected data is needed to generalize results. That being said, the number of patients included and analyzed in this study offers a measure of diagnostic precision and clinical utility of using molecular test for thyroid nodules.

\section{CONCLUSION}

Select molecular mutations in thyroid nodules were associated with specific Bethesda diagnostic categories on cytology. BRAF V600E mutations were mostly associated with Bethesda categories V and VI, whereas RAS and EIF1AX mutations, copy number alterations, and GEP were related to Bethesda categories III and IV. This information may guide clinicians in the prediction of molecular mutations of thyroid nodules and potentially improve management.

\section{REFERENCES}

1. Hall SF, Walker H, Siemens R, Schneeberg A. Increasing detection and increasing incidence in thyroid cancer. World journal of surgery: official journal of the international society of surgery/societe internationale de chirurgie . 2009;33(12):2567-2571. doi:10.1007/s00268-009-0226-9

2. Sosa J, Hanna J, Robinson K, Lanman, R. (2013). Increases in thyroid nodule fine-needle aspirations, operations, and diagnoses of thyroid cancer in the united states. Surgery, 154 (6), 1420-6. doi:10.1016/j.surg.2013.07.006

3. Lewis C, Chang K, Pitman M, Faquin W, Randolph G. (2009). Thyroid fine-needle aspiration biopsy: Variability in reporting. Thyroid: Official Journal of the American Thyroid Association,19 (7), 717-23. doi:10.1089/thy.2008.0425

4. Cibas ES, Ali SZ. The 2017 Bethesda system for reporting thyroid cytopathology. Thyroid. 2017;27(11):1341-1346.

5. Haugen BR, Alexander EK, Bible KC, Doherty GM, Mandel SJ, Nikiforov YE, Pacini F, Randolph GW, Sawka AM, Schlumberger M, Schuff KG, Sherman SI, Sosa JA, Steward DL, Tuttle RM, Wartofsky L. 2015 American Thyroid Association Management Guidelines for Adult Patients With Thyroid Nodules and Differentiated Thyroid Cancer: The American Thyroid Association Guidelines Task Force on Thyroid Nodules and Differentiated Thyroid Cancer. Thyroid. 2016. Jan;26(1):1-133. doi: 10.1089/thy.2015.0020

6. Varshney R, Forest V-I, Mascarella MA, Zawawi F, Rochon L, Hier MP, Mlynarek A, Tamilia M, Payne RJ. The mcgill thyroid nodule score - does it help with indeterminate thyroid nodules? Journal of otolaryngology - head \& neck surgery . 2015;44(1). doi:10.1186/s40463-015-0058-6

7. Valderrabano P, Khazai L, Thompson Z, Leon M, Otto K, Hallanger-Johnson J, Wadsworth JT, Wenig BM, Chung CH, Centeno BA, McIver B. (2017). Cancer risk stratification of indeterminate thyroid nodules: A cytological approach. Thyroid: Official Journal of the American Thyroid Association,27 (10), 1277-1284. doi:10.1089/thy.2017.0221

8. Mehta V, Nikiforov Y, Ferris RL. (2013). Use of molecular biomarkers in fna specimens to personalize treatment for thyroid surgery. Head $\mathscr{E}$ Neck, 35 (10), 1499-506. doi:10.1002/hed.23140

9. Bhaijee F, Nikiforov YE. Molecular analysis of thyroid tumors. Endocrine pathology . 2011;22(3):126133. doi:10.1007/s12022-011-9170-y

10. Krasner JR, Alyouha N, Pusztaszeri M, Forest VI, Hier MP, Avior G, Payne RJ. Molecular mutations as a possible factor for determining extent of thyroid surgery. J Otolaryngol Head Neck Surg . 2019;48(1):17. doi:10.1186/s40463-019-0372-5

11. Nikiforova MN, Kimura ET, Gandhi M, Biddinger PW, Knauf JA, Basolo F, Zhu Z, Giannini R, 
Salvatore G, Fusco A, Santoro M, Fagin JA, Nikiforov YE. Braf mutations in thyroid tumors are restricted to papillary carcinomas and anaplastic or poorly differentiated carcinomas arising from papillary carcinomas. The journal of clinical endocrinology and metabolism . 2003;88(11):5399-5404.

12. Kim TH, Park YJ, Lim JA, Ahn HY, Lee EK, Lee YJ, Kim KW, Hahn SK, Youn YK, Kim KH, Cho BY, Park DJ. The association of the braf(v600e) mutation with prognostic factors and poor clinical outcome in papillary thyroid cancer: a meta-analysis. Cancer . 2012;118(7):1764-1773. doi:10.1002/cncr.26500

13. Elisei R, Viola D, Torregrossa L, Giannini R, ROmei C, Ugolini C, Molinaro E, Agate L, Biagini A, Lupi C, Valerio L, Materazzi G, Miccoli P, Piaggi P, Pinchera A, Vitti P, Basolo F. The braf(v600e) mutation is an independent, poor prognostic factor for the outcome of patients with low-risk intrathyroid papillary thyroid carcinoma: single-institution results from a large cohort study. The journal of clinical endocrinology and metabolism . 2012;97(12):4390-4398. doi:10.1210/jc.2012-1775

14. Li C, Lee KC, Schneider EB, Zeiger MA. Braf v600e mutation and its association with clinicopathological features of papillary thyroid cancer: a meta-analysis. The journal of clinical endocrinology and metabolism . 2012;97(12):4559-4570. doi:10.1210/jc.2012-2104

15. Xing M, Westra WH, Tufano RP, B Cohen Y, Rosenbaum E, Rhoden KJ, Carson KA, Vasko V, Larin A, Tallini G, Tolaney S, Holt EH, Hui P, Umbricht CB, Basaria S, Ewertz M, Tufaro AP, Califano JA, Ringel MD, Zeiger MA, Sidransky D, Ladenson PW. Braf mutation predicts a poorer clinical prognosis for papillary thyroid cancer. The journal of clinical endocrinology and metabolism . 2005;90(12):63736379 .

16. Celik M, Bulbul BY, Ayturk S, Durmus Y, Gurkan H, Can N, Tastekin E, Ustun F, Sezer A, Guldiken S.The relation between brafv600e mutation and clinicopathological characteristics of papillary thyroid cancer. Medicinski glasnik : official publication of the medical association of zenica-doboj canton, bosnia and herzegovina . 2020;17(1). doi:10.17392/1086-20

17. Adeniran AJ, Zhu Z, Gandhi M, Steward DL, Fidler JP, Giordano TJ, Biddinger PW, Nikiforov YE. Correlation between genetic alterations and microscopic features, clinical manifestations, and prognostic characteristics of thyroid papillary carcinomas. The american journal of surgical pathology . 2006;30(2):216-222.

18. Patel SG, Carty SE, McCoy KL, Ohori NP, LeBeau SO, Seethala R, Nikiforova MN, Nikiforov YE, Yip L. Preoperative detection of ras mutation may guide extent of thyroidectomy. Surgery . 2017;161(1):168-175. doi:10.1016/j.surg.2016.04.054

19. Guan H, Toraldo G, Cerda SR, Godley FA, Rao SR, McAneny D, Doherty G, Braverman L, Lee SL. Utilities of ras mutations in pre-operative fine needle biopsies for decision-making for thyroid nodule management: results from a single-center prospective cohort. Thyroid: official journal of the american thyroid association . 2020;2020 Jan 29. doi:10.1089/thy.2019.0116

20. Patel KN, Yip L, Lubitz CC, Grubbs EG, Miller BS, Shen W, Angelos P, Chen H, Doherty GM, Fahey TJ, Kebebew E, Livolsi VA, Perrier ND, Sipos JA, Sosa JA, Steward D, Tufano RP, McHenry CR, Carty $\mathrm{SE}$. The american association of endocrine surgeons guidelines for the definitive surgical management of thyroid disease in adults. Annals of surgery . 2020;271(3):93. doi:10.1097/SLA.0000000000003580

21. Nikiforov YE, Ohori NP, Hodak SP, Carty SE, LeBeau SO, Ferris RL, Yip L, Seethala RR, Tublin ME, Stang MT, Coyne C, Johnson JT, Steward AF, Nikiforova MN. Impact of mutational testing on the diagnosis and management of patients with cytologically indeterminate thyroid nodules: a prospective analysis of 1056 fna samples. The journal of clinical endocrinology and metabolism . 2011;96(11):33903397. doi:10.1210/jc.2011-1469

22. Muzza M, Colombo C, Pogliaghi G, Karapanou O, Fugazzola L. Molecular markers for the classification of cytologically indeterminate thyroid nodules. Journal of endocrinological investigation. 2019;2019 Dec 18. doi:10.1007/s40618-019-01164-w

23. Alexander EK, Kennedy GC, Baloch ZW, Cibas ES, CHudova D, Diggans J, Friedman L, Kloos RT, Livolsi VA, Mandel SJ, Raab SS, Rosai J, Steward DL, Walsh PS, Wilde JI, Zeiger MA, Lanman RB, Haugen BR. Preoperative diagnosis of benign thyroid nodules with indeterminate cytology. The new england journal of medicine . 2012;367(8):705-715. doi:10.1056/NEJMoa1203208

24. Roth MY, Witt RL, Steward DL. Molecular testing for thyroid nodules: review and current state. Can- 
cer . 2018;124(5):888-898. doi:10.1002/cncr.30708

25. Huy Gia Vuong, Uyen N P Duong, Ahmed M A Altibi, Ngo HTT, Pham TQ, Tran HM, Gandolfi G, Hassell L. A meta-analysis of prognostic roles of molecular markers in papillary thyroid carcinoma. Endocrine connections . 2017;6(3):17. doi:10.1530/EC-17-0010
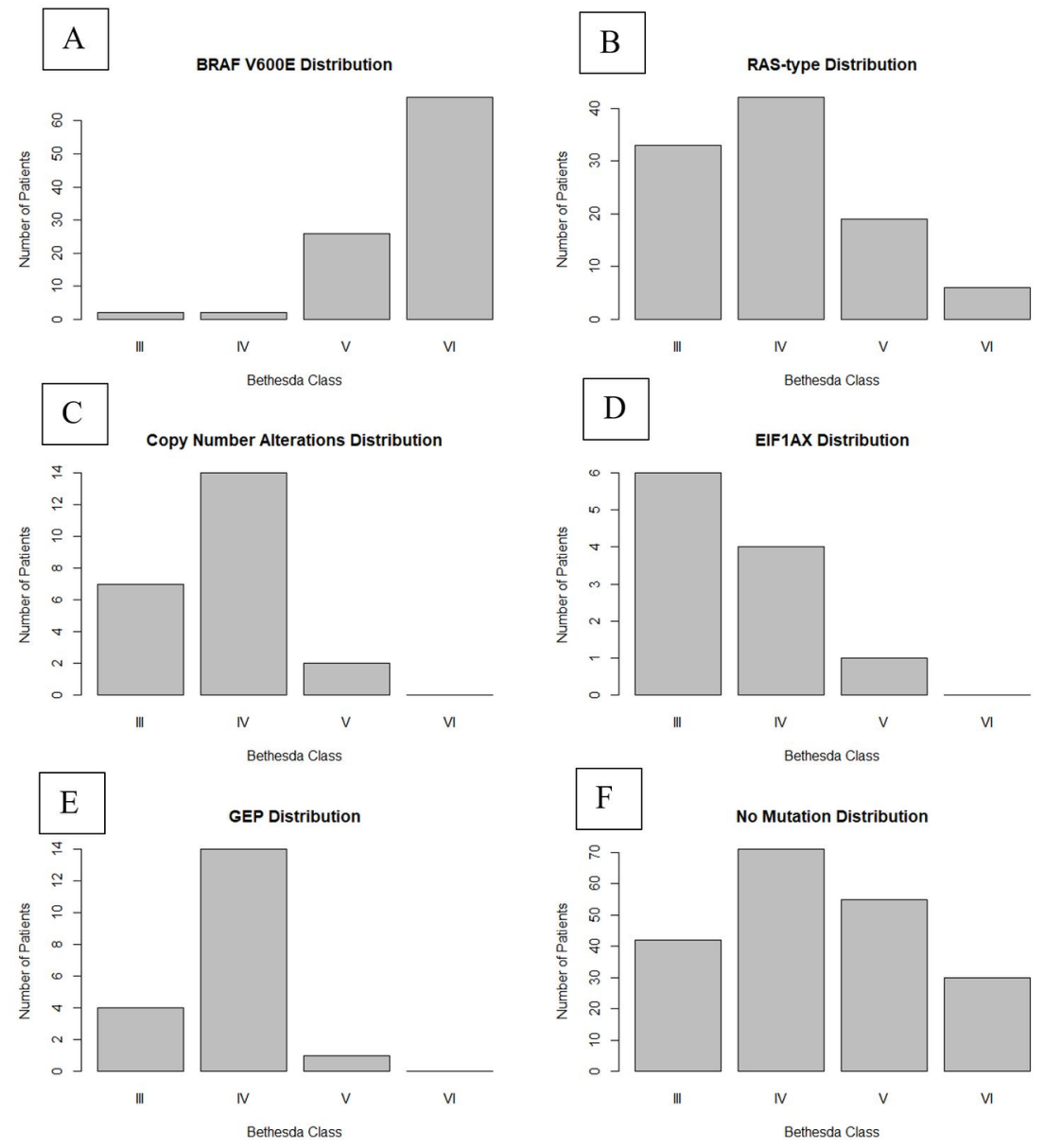

\section{Hosted file}

newTABLE1.pdf available at https://authorea.com/users/402997/articles/514682-association-ofbethesda-category-and-molecular-mutation-in-patients-undergoing-thyroidectomy

\section{Hosted file}

newTABLE2 .pdf available at https://authorea.com/users/402997/articles/514682-association-ofbethesda-category-and-molecular-mutation-in-patients-undergoing-thyroidectomy

\section{Hosted file}

newTABLE3.pdf available at https://authorea.com/users/402997/articles/514682-association-ofbethesda-category-and-molecular-mutation-in-patients-undergoing-thyroidectomy 